\title{
Polyphyllin I suppresses proliferation and promotes apoptosis of gastric cancer cell by inhibiting stat3 phosphorylation
}

\author{
Jun Han $^{1}$, Jie Kan ${ }^{1}$, Xinguo Wang ${ }^{1}$, Qingling Yuan ${ }^{1}$, Xiaoyan Song ${ }^{1}$, Min Huang ${ }^{2}$, Hao Wu ${ }^{1,3}$ \\ ${ }^{1}$ Department of Oncology, Qinghai Provincial People's Hospital, Xining, China; ${ }^{2}$ Department of Geriatrics, ${ }^{3}$ Department of Oncology, The First \\ Affiliated Hospital of Nanjing Medical University, Nanjing, China \\ Contributions: (I) Conception and design: H Wu; (II) Administrative support: M Huang; (III) Provision of study materials or patients: J Han, J Kan; (IV) \\ Collection and assembly of data: X Song; (V) Data analysis and interpretation: X Wang, Q Yuan; (VI) Manuscript writing: All authors; (VII) Final \\ approval of manuscript: All authors. \\ Correspondence to: Hao Wu. Department of Oncology, The First Affiliated Hospital of Nanjing Medical University, 300 Guangzhou Road, Nanjing \\ 210029, China. Email: whdactor@163.com.
}

\begin{abstract}
Background: Polyphyllin I, a bioactive phytochemical extracted from Rhizoma Paridis, has been reported to exhibit anticancer activity. However, little is known about the potential of Polyphyllin I in induction of gastric cancer (GC) cell apoptosis and its underlying mechanisms.

Methods: Dual-luciferase reporter assay was performed to test the bioactivity of Polyphyllin I on inhibiting JAK2/STAT3 signaling pathway. The anti-proliferation activity of Polyphyllin I was tested using cell clone formation assay. The effect of Polyphyllin I on cell cycle and apoptosis were confirmed by flow cytometry and TUNEL assay. Western blot was used to test the effect of Polyphyllin I on JAK/STAT3 pathway and apoptosis related proteins. The subcutaneous GC mouse model was established to examine whether polyphyllin I could inhibit GC growth in vivo.

Results: A dual-luciferase reporter assay showed that polyphyllin I could inhibit the activity of Bcl-2 which is downstream of JAK2/STA3. Polyphyllin I significantly inhibited GC cell proliferation and induced cell apoptosis in a dose-dependent manner. The western blot results indicated that polyphyllin I mainly inhibited the phosphorylation of STAT3 by the way which is different from AG490. It was inferred that polyphyllin I may inhibit the JAK2/STAT3 pathway and affect the expression level of apoptosis related genes. Finally, in the tumor xenograft experiment proved that polyphyllin I significantly inhibited the growth of GC in vivo.

Conclusions: Polyphyllin I may play its anticancer activity by inhibiting phosphorylation of STAT3 in GC cells.
\end{abstract}

Keywords: Gastric cancer (GC); JAK2/STAT3; polyphyllin I; STAT3 inhibitor; apoptosis

Submitted Jan 01, 2020. Accepted for publication Jun 19, 2020.

doi: $10.21037 /$ tcr-20-66

View this article at: http://dx.doi.org/10.21037/tcr-20-66

\section{Introduction}

Gastric cancer (GC) is a type of highly malignant lesion of the digestive tract that usually occurs in middle-aged and elderly individuals. The latest statistics show that GC ranks fourth out of all malignant tumors in incidence and third in mortality rates (1). Over the past 50 years, clinicians have been using traditional treatments, such as surgery, radiation therapy, chemotherapy, and combination therapy, as the main treatments for cancer, but with little success.
Therefore, the improvement of GC prognosis and the development of new anticancer drugs is imperative.

The JAK2/STAT3 signaling pathway participates in physiological and pathological reactions, cell proliferation, differentiation, apoptosis, and other processes $(2,3)$. An abnormally sustained activation of STAT3 was identified (4-6) in breast, lung, head and neck cancer, among others. Previous studies have shown that the JAK2/STAT3 pathway affects cancer development by influencing 
tumor cell survival, proliferation, apoptosis, invasion and migration $(7,8)$. The abnormal activation of the JAK2/ STAT3 signaling pathway, particularly the abnormal phosphorylation of the STAT3 protein and mutation of the STAT3 gene, plays a key role in tumor formation. Several study results have shown that JAK2/STAT3 pathway inhibitors, such as AG490, could be powerful at killing cancer and improving patient survival (9-11). Therefore, the inhibition of STAT3 phosphorylation through direct and indirect mechanisms could be an important approach to cancer treatment.

Rbizoma Paridis is a common Chinese herbal medicine that mainly grows in China. Polyphyllin I is one of the most important active components extracted from Rhizoma Paridis. Previous studies have shown that polyphyllin I exerts a good inhibitory effect on multiple types of tumor cells, such as liver, breast and lung cancer, and osteosarcoma cells. The underlying mechanism of this effect may be associated with the cytotoxicity of polyphyllin I and its role in accelerating apoptosis, affecting the tumor cell cycle, inhibiting the generation of the tumor vasculature and regulating immune function (12-16). However, the antitumor mechanism of polyphyllin I in GC is not particularly clear. In the present study, it was found that polyphyllin I had an inhibitory effect on the activity of STAT3 phosphorylation in GC cells. The primary purpose of the present study was to investigate the antitumor mechanism of polyphyllin I on GC.

We present the following article in accordance with the ARRIVE reporting checklist (available at http://dx.doi. org/10.21037/tcr-20-66).

\section{Methods}

Cell culture and compound dilution. AGS and SGC7901 GC cell lines and GES-1 (Human Gastric mucosal Epithelial Cells) were acquired from American Type Culture Collection and cultured in a $37^{\circ} \mathrm{C}$ thermostatic incubator (95\% air, 5\% $\mathrm{CO}_{2}$ ) with Gibco RPMI-1640 medium with $10 \%$ FBS. Polyphyllin $\mathrm{I}$ is the main active ingredient of Rbizoma Paridis. The polyphyllin I powder used was purchased from BioChemPartner (Shanghai, China) and diluted with DMSO. The primary working concentration was $0.5 \mu \mathrm{M}$.

\section{Treatment of GC cells with polyphyllin I, IL-6 and AG490}

AGS GC cells were cultured in a $37^{\circ} \mathrm{C}$ thermostatic incubator $\left(95 \%\right.$ air, $5 \% \mathrm{CO}_{2}$ ) with conditional medium for $>48 \mathrm{~h}$. The $\mathrm{IC}_{50}$ of the drug was calculated by measuring cell activity at different drug concentrations. The conditional medium consisted of Gibco RPMI-1640 medium, $10 \% \mathrm{FBS}, 1 \%$ of the double resistance and the appropriate concentration of the drug including polyphyllin I $(1$ and $2 \mu \mathrm{M})$, IL-6 $(100 \mu \mathrm{g} / \mathrm{L})$ or AG490 $(30 \mu \mathrm{M})$. The concentration of the drug used in the following experiments was selected based on the $\mathrm{IC}_{50}$ value.

\section{Dual-luciferase reporter assay}

The promoter fragment of the $B c l-2$ gene was constructed into PGL3 plasmid and named PGL3-Bcl2-Luc. Renilla (phRL-TK) plasmid was purchased from Promega Corporation (Madison, WI, USA). PGL3-Bcl2-Luc (15 $\mu \mathrm{g})$ and Renilla plasmid $(1 \mu \mathrm{g})$ were co-transfected into GES1 cells with $30 \mathrm{uL}$ Lipo2000 transfection reagents. The medium was refreshed after $6 \mathrm{~h}$ and cells were cultured for 12 h. On day 2, GES-1 cells were trypsinized and seeded into 24-well culture plates at a density of 50,000 cells/well in growth medium supplemented with $10 \%$ serum. Following culture for $36 \mathrm{~h}$, cells were lysed and the luciferase activity was measured using Dual-Luciferase ${ }^{\circledR}$ Reporter Assay System (Promega, Corporation). All experiments were conducted three times.

\section{Western blot analysis}

Treated AGS GC cells were collected and lysed with RIPA lysis buffer (Epizyme; MA, USA; PC101) containing protease inhibitors. The protein concentration was measured by Bicinchoninic Acid Protein Assay Kit (Epizyme; ZJ101). Equal amounts of protein were separated on sodium dodecyl sulfate polyacrylamide gel electrophoresis (Epizyme; PG110) and then transferred to polyvinylidene difluoride membranes (EMD Millipore, Billerica, USA). The membranes were blocked with $1 \mathrm{x}$ blocking buffer (Epizyme; PS108) for 30 mins, followed by incubation with primary antibodies at $4{ }^{\circ} \mathrm{C}$ for $12 \mathrm{~h}$. The following primary antibodies were used: p-JAK1 (Cell Signaling Technology, Inc., Danvers, MA, USA; 3331), JAK1 (Cell Signaling Technology Inc.; 3344), p-STAT1 (Cell Signaling Technology, Inc.; 8826), STAT1 (Cell Signaling Technology, Inc.; 9175), p-JAK2 (Cell Signaling Technology, Inc., Danvers, MA, USA; 3771), JAK2 (Cell Signaling Technology Inc.; 3230), p-STAT3 (Cell Signaling Technology, Inc.; 9145), STAT3 (Cell Signaling Technology, 
Inc.; 9139), Bax (Protein Tech Group, Inc., Chicago, IL, USA; 50599), Bcl-2 (ProteinTech Group, Inc.; 12789), Bad (ProteinTech Group, Inc.; 10435), Bcl-XL (ProteinTech Group, Inc.; 10783), FasL( ProteinTech Group, Inc.; 13098), TNFa( ProteinTech Group, Inc.; 17590), GAPDH (Protein Tech Group, Inc.; 10494). Following incubation with secondary-HRP-antibodies (ProteinTech, Group, Inc.; SA00001) for $2 \mathrm{~h}$ at room temperature, the signal on the membranes was visualized using an enhanced chemiluminescence kit (Epizyme; SQ201). Images of the protein bands were captured using Tanon 5200 Biotanon and the results were analyzed using image $\mathrm{J}$ software.

\section{Cell proliferation assay}

Two kinds of human GC cell lines (AGS and SGC7901) were used for the cell proliferation assay. A total of 5,000 cells/well were seeded into 96-well plates and treated with different concentrations of polyphyllin I ( 1 and $2 \mu \mathrm{M})$ for $48 \mathrm{~h}$. The cell proliferation ability was examined using a Cell Counting Kit-8 (Dojindo Molecular Technologies, Inc., Kumamoto, Japan), following the manufacturer's instructions.

\section{Clonogenic assay}

Two types of human GC cell lines (AGS and SGC7901) were used for the clonogenic assay. A total of 500 cells/well were seeded into 6-well plates and treated with different concentrations of polyphyllin I ( 1 and $2 \mu \mathrm{M})$ for $48 \mathrm{~h}$. Every other day, the medium was refreshed with the corresponding concentration of the drug. Cells were continuously cultured for 15 days, until single cell had grown into a colony. Next, the medium was removed and cells were fixed with 4\% PFA for $20 \mathrm{~min}$. Following natural air drying and staining with $0.5 \%$ crystal violet solution for $1 \mathrm{~h}$, images were captured.

\section{Apoptosis assay}

Two types of human GC cells (AGS and SGC7901) were seeded into 6-well plates. Following treatment with different concentrations of polyphyllin I ( 1 and $2 \mu \mathrm{M})$ for $48 \mathrm{~h}$, cells were digested with trypsin and washed three times with cold PBS. Unfixed cells were stained with Annexin V/PI kit (BD Biosciences, Tokyo, Japan), following the manufacturer's instructions. Subsequently, cells were subjected to flow cytometric analysis with FACSCalibur (BD Biosciences).

\section{Cell cycle assay}

Two types of human GC cells (AGS and SGC7901) were seeded into 6-well plates. Following treatment with different concentrations of polyphyllin I $(1$ and $2 \mu M)$ for $48 \mathrm{~h}$, cells were digested with trypsin and washed three times with cold PBS. Cells were fixed with $70 \%$ ethanol at $4{ }^{\circ} \mathrm{C}$ for $12 \mathrm{~h}$ and washed with PBS 3 times. Next, cells were digested by RNase A at $37^{\circ} \mathrm{C}$ for $30 \mathrm{~min}$ and stained with PI following the manufacturer's instructions. Subsequently, cells were subjected to flow cytometric analysis by FACSCalibur (BD Biosciences).

\section{TUNEL assay}

Two types of human GC cells (AGS and SGC7901) were seeded into 6-well plates. Following treatment with different concentrations of polyphyllin I $(1$ and $2 \mu \mathrm{M})$ for $48 \mathrm{~h}$, cells were fixed with $4 \% \mathrm{PFA}$ for $30 \mathrm{~min}$, and washed with PBS for 3 times. Add PBS containing $0.3 \%$ Triton X-100 and incubated at room temperature for 5 min, and wash with PBS for 3 times. Prepare appropriate amount of TUNEL detection solution and incubate at $37^{\circ} \mathrm{C}$ in dark for $60 \mathrm{~min}$, wash with PBS for 3 times. Finally, the section was stained with DAPI and sealed for microscopic observation.

\section{Immunobistochemistry}

Formalin-fixed paraffin-embedded sections $(10 \mu \mathrm{M})$ were prepared. They were deparaffinized in xylene, rehydrated and incubated in $30 \% \mathrm{H}_{2} \mathrm{O}_{2}$. The sections were then incubated with primary ki-67 antibody (Protein Tech Group, Inc.; 27309) for $12 \mathrm{~h}$ at $4{ }^{\circ} \mathrm{C}$. Proteins were detected using light microscopy.

\section{Tumor-bearing nude mice}

AGS cells $\left(5 \times 10^{6}\right.$, pre-mixed with Matrigel prior to injection) were injected into the back of the right side of 6-week old male BALB/cA nude mice. When the subcutaneous tumor block reached a certain volume $\left(\geq 100 \mathrm{~mm}^{3}\right)$, the nude mice were randomly divided into two groups ( $\mathrm{n}=4$ /group), the control (PBS; twice a week) and polyphyllin I dosing $(0.2 \mathrm{mg} / \mathrm{kg}$; twice a week) groups. The intraperitoneal injection was administered every 4 days for 4 weeks, to measure the change in solid tumor volume and weight following treatment. Tumor volumes 
were calculated according to the recognized formula: $\mathrm{V}=$ $\left(\right.$ length $\times$ width $\left.^{2}\right) / 2$. The length and width of tumors in live mice were measured using a Vernier caliper. Finally, mice were sacrificed, tumors samples were excised and the tissue weight was measured. Experiments were performed under a project license (NO.: 2018-SRFA-088) granted by Institutional Ethics Board of The First Affiliated Hospital of Nanjing Medical University, in compliance with Nanjing Medical University institutional guidelines for the care and use of animals.

\section{Statistical analysis}

All data are presented as the mean $\pm \mathrm{SD}$ of three independent experiments. GraphPad Prism 6 software was used for statistical analysis. Significant differences between two groups were determined using student's $t$-test, and among multiple groups using one- or two-way ANOVA. The level of significance was set at $\mathrm{P}<0.05, \mathrm{P}<0.01$ and $\mathrm{P}<0.001$, where $\mathrm{P}<0.05$ was considered to indicate a statistically significant difference.

\section{Results}

\section{Polyphyllin I can inbibit the activity of GC cells}

Polyphyllin I is one of the most important active components extracted from Rhizoma Paridis and has an anticancer effect (Figure 1A). In order to examine the inhibitory effect of polyphyllin I on GC cell growth, a reporter gene test system was constructed. The system used the plasmid pGL3-Bcl2-Luc (containing transcription activator and luciferase) and internal plasmid Renilla (phRL-TK) to co-transfect GES-1 cells. Known JAK2/ STAT3 signaling pathway agonist IL-6 and inhibitor AG490, which can regulate the expression level of Bcl-2, were used to test the stability and reliability of this system (Figure 1B). The results showed that the system was feasible.

In order to determine the optimal concentration of polyphyllin I, $10 \mu \mathrm{M}$ were selected as a preliminary screening concentration. Further reduced concentrations, however, $(5,2,1$ and $0.5 \mu M)$ still had an inhibitory effect (Figure $1 B$ ). To promote polyphyllin I in clinical research and application, we studied the mechanism of its inhibitory activity on GC cells. The appropriate treatment concentrations of polyphyllin I used in the following experiments were designed according to the $\mathrm{IC}_{50}$ value (Figure 1). The $\mathrm{IC}_{50}$ of polyphyllin I in AGS and SGC7901 cells was 2.080 and $1.905 \mu \mathrm{M}$, respectively. In order to maintain a good cellular status, concentrations of 1 and 2 $\mu M$ were used.

\section{Polyphyllin I can inbibit the IL-6-induced p-STAT3 upregulation}

The JAK2/STAT3 signaling pathway mainly consists of three components, cytokine receptors, JAK2, and STAT3. Activated JAK2 induces the phosphorylation of STAT3. p-STAT3 combines with the corresponding target gene promoter and activates the corresponding gene transcription and expression. It was shown that polyphyllin I inhibit the phosphorylation of STAT3 in GC AGS cells in a concentration-dependent manner. The expression of p-JAK2 was not affected in the AGS GC cells treated with $1 \mu M$ polyphyllin I. However, as the drug concentration increased $(5 \mu \mathrm{M})$, the p-JAK2 expression began to decrease (Figure 2A). IL-6, a key proinflammatory cytokine, is involved in a spectrum of diseases, including cancer. The released IL-6 preferentially activates STAT3 through the phosphorylation of Tyr705 via the JAK signaling pathway. IL-6 treatment can increase the phosphorylation of JAK2 and STAT3. In the present study, the $\mathrm{IC}_{50}$ of IL-6 on AGS was calculated to be $107.8 \mathrm{ng} / \mathrm{mL}$, and $100 \mathrm{ng} / \mathrm{mL}$ was selected as the optimal concentration (Figure 1D). Western blot analysis results proved that the lower concentration of polyphyllin I $(1 \mu \mathrm{M})$ can offset the IL-6-induced increased p-STAT3 expression $(100 \mathrm{ng} / \mathrm{mL})$. A series of different concentrations of IL- 6 was involved in the screening system, proving that polyphyllin I can block the effect of IL-6 on Bcl-2 (Figure 1B). However, the IL-6 $(100 \mathrm{ng} / \mathrm{mL})$ induced high expression level of p-JAK2 was not affected by polyphyllin $\mathrm{I}(1 \mu \mathrm{M})$ (Figure $2 B)$. These results indicated that polyphyllin I mainly affected the phosphorylation of STAT3 and did not influence the phosphorylation of JAK2 at low concentrations, such as $1 \mu \mathrm{M}$.

\section{Polyphyllin I may work as a STAT3 inbibitor}

It is known that the main function of AG490 is to inhibit JAK2 phosphorylation. AGS GC cells were treated with AG490 and polyphyllin I to determine if they interact with each other. The concentration of AG490 used in the following studies was $30 \mu \mathrm{M}$, which was designed based on the $\mathrm{IC}_{50}$ value (Figure 1D). The expression level of p-JAK2 and p-STAT3 was detected by western blot analysis. The results showed that polyphyllin I $(1 \mu M)$ did not affect 


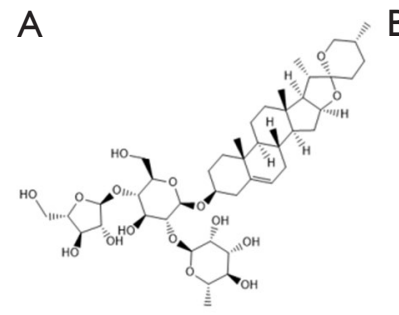

C
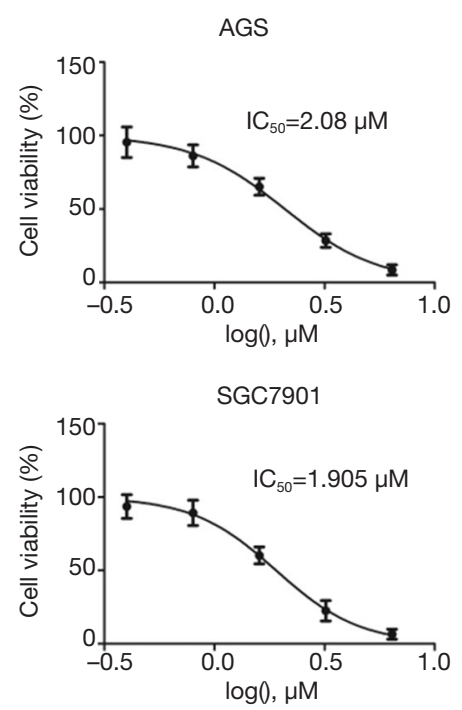
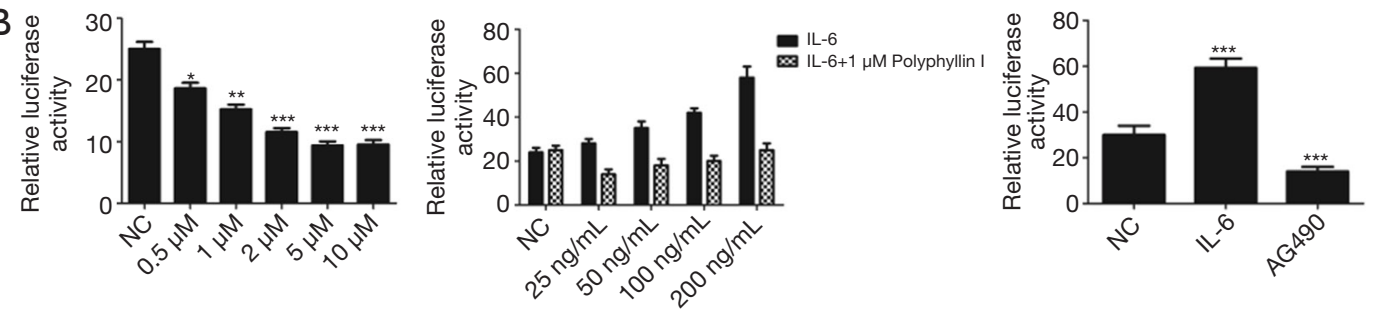

D

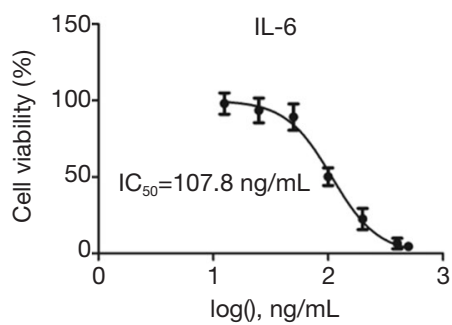

SGC7901
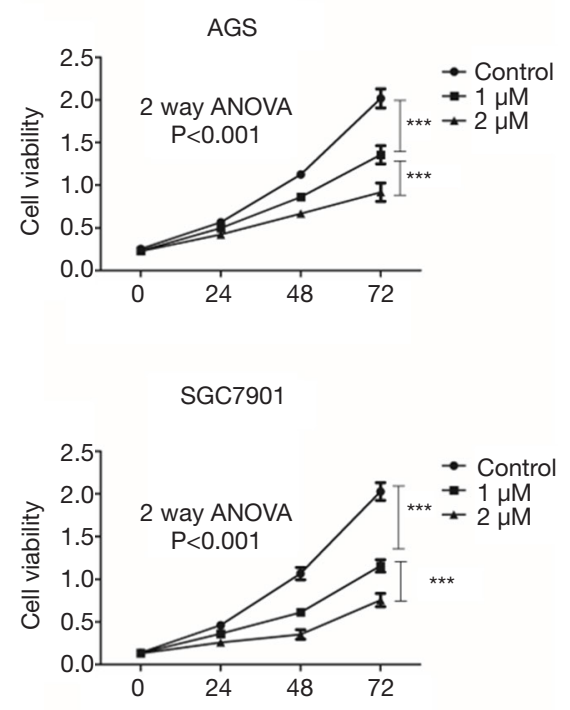

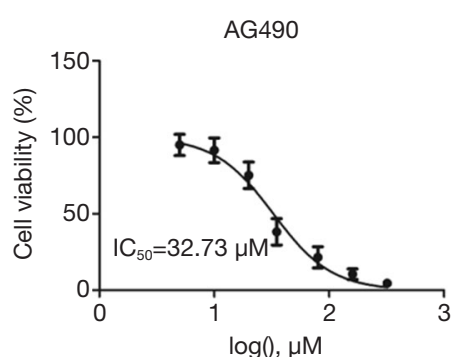

Figure 1 Polyphyllin I can inhibit the activity of GC cells. (A) Molecular structure of polyphyllin I; (B) stability and reliability of the screening system by dual-luciferase reporter assay; (C) design of best polyphyllin I concentration for the experiments (n>3); (D) design of best IL-6 and AG490 concentration for the experiments $(\mathrm{n}>3)$. Error bars represent the mean \pm SD of three independent experiments. *, $\mathrm{P}<0.05 ;{ }^{* *}, \mathrm{P}<0.01{ }^{* * *}, \mathrm{P}<0.001$ vs. control group $(\mathrm{n}>3)$. Significant differences between the control and drug groups were determined using two-way ANOVA with a $\mathrm{P}<0.001$. IL-6 is interleukin-6, AG490 is a tyrosine kinase inhibitor that inhibits Stat-3 and JAK2/3.

the p-JAK2 expression, the target of AG490 in GC cells (Figure 2C). The results indicated that the way polyphyllin I worked was indeed different from that of AG490. We therefore inferred that polyphyllin I might act on STAT3 and work as a STAT3 inhibitor. Since polyphyllin I is worked as an inhibitor of STAT3, does it also inhibit JAK1/STAT1 pathway? We then examined the expression of JAK1, p-JAK1, STAT1 and p-STAT1 in cells treated with the polyphyllin I. The results showed that polyphyllin I can also inhibit the phosphorylation of JAK1 and STAT1 (Figure 2D).

\section{Polyphyllin I inbibit the proliferation and promotes the apoptosis of GC cells in vitro}

In tumors, products of the JAK2/STAT3 signaling pathway target genes are mainly associated with the proliferation and survival of cancer cells. Therefore, two different human GC cell lines (AGS and SGC7901) were selected to detect the inhibitory activity of polyphyllin I on GC cell proliferation and clone formation assay. The experimental results showed that polyphyllin I exerted a significant inhibitory effect on the proliferation (Figure 1C) and clone formation assay of both tested GC cell lines (Figure 3A). Cell cycle analysis demonstrated that the G2/M fraction increased and the $\mathrm{S}$ fraction decreased in AGS cells, following treatment with 1 and $2 \mu \mathrm{M}$ polyphyllin I (Figure $3 B$ ). However, no significant change in the G0-G1 phase was observed between the drug and control groups. Cell cycle disturbance may lead to necrosis and apoptosis, which can be detected by flow cytometry.

Subsequently, the effect of polyphyllin I on the promotion of apoptosis was detected in AGS and SGC7901 
A
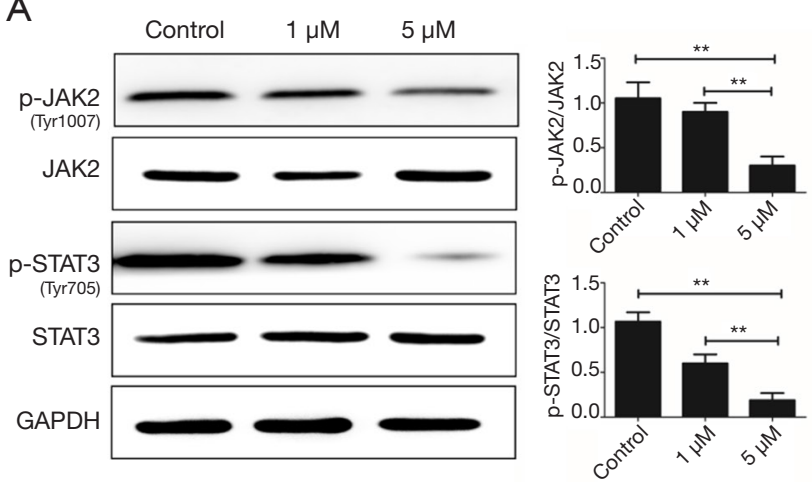

C

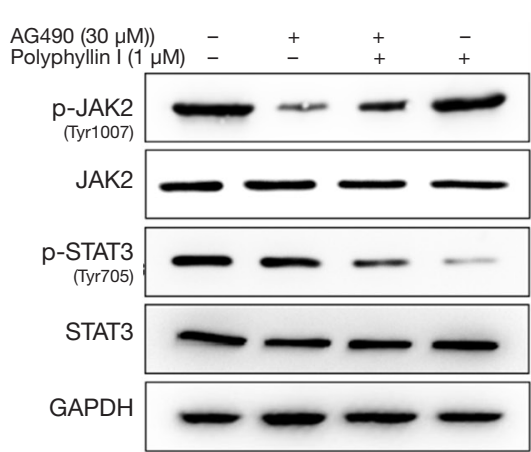

B

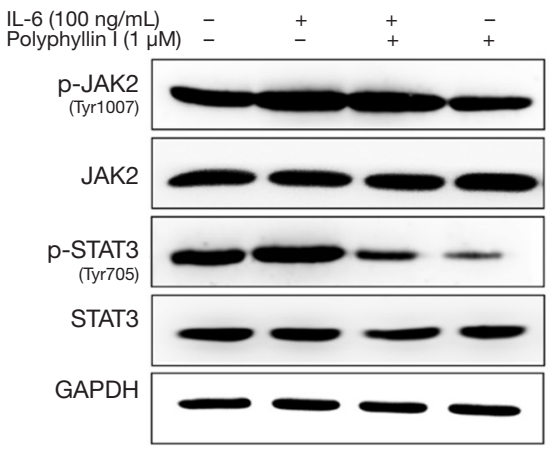

D

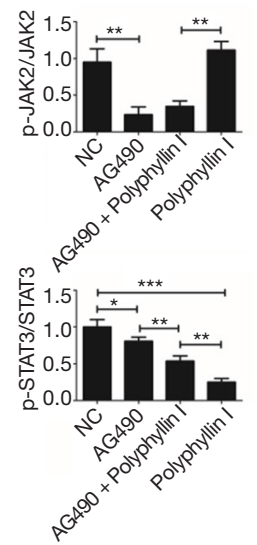

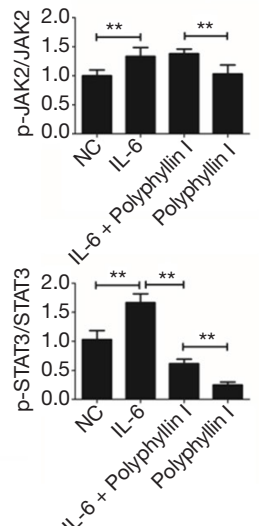
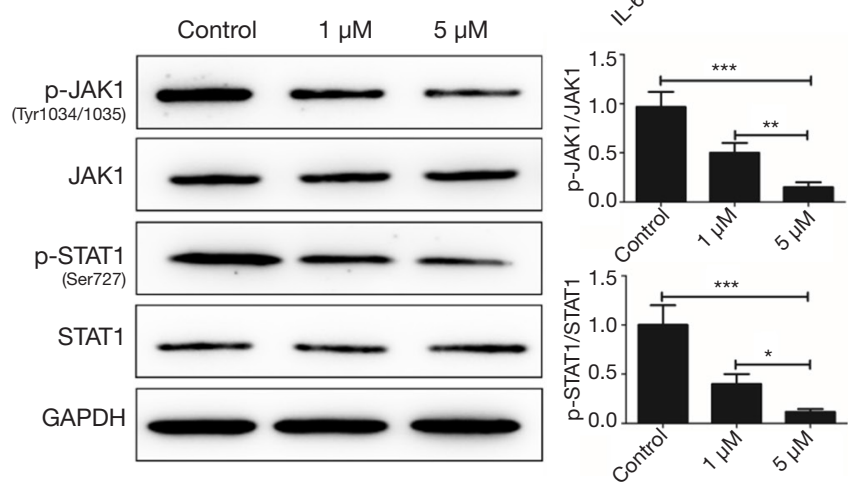

Figure 2 Polyphyllin I may work as a STAT3 inhibitor. (A) Western blot analysis of phosphorylated JAK2 (Tyr1007) and STAT3 (Tyr705) in GC cells treated with 1 and $5 \mu \mathrm{M}$ polyphyllin I for $48 \mathrm{~h}$; (B) Western blot analysis of phosphorylated JAK2 (Tyr1007) and STAT3 (Tyr705) in GC cells treated with polyphyllin I and IL-6 for 48 h; (C) Western blot analysis of phosphorylated JAK2 (Tyr 1007) and STAT3 (Tyr705) in GC cells treated with polyphyllin I and AG490 for 48 h; (D) Western blot analysis of phosphorylated JAK1 (Tyr1034/1035) and STAT1 (Ser727) in GC cells treated with 1 and $5 \mu \mathrm{M}$ polyphyllin I for $48 \mathrm{~h}$. Error bars represent the mean \pm SD of three independent experiments. *, $\mathrm{P}<0.05 ;{ }^{* *}, \mathrm{P}<0.01 ;{ }^{* * *}, \mathrm{P}<0.001$ vs. control group $(\mathrm{n}>3)$.

by flow cytometry. The rates of early and late apoptosis of AGS cells in the drug group were 3-6 times higher than those in the control. The results showed that polyphyllin I obviously promoted apoptosis of GC cells (Figure 3C). TUNEL assay also confirmed the pro-apoptotic effect of polyphyllin I on GC cells (Figure 3D).

In addition, the effect of polyphyllin I on any apoptosisrelated proteins was also detected by WB assay. The results confirmed that polyphyllin I can not only activate death factor-mediated apoptosis by increasing the expression of pro-apoptotic factors (FasL, TNFa), but also activate mitochondrial pathway mediated apoptosis by inducing pro-apoptotic factors (Bax, Bad). Moreover, the drug can inhibit the expression of some anti-apoptotic factors (bcl-2, bcl-xl) (Figure 4). The apoptosis detection and western blot analysis suggested that polyphyllin I could promote GC cell apoptosis through both death receptor and mitochondrial pathways.

\section{Polyphyllin I inbibit GC growth in vivo}

The subcutaneous GC mouse model was established to examine whether polyphyllin I could inhibit GC growth in vivo. First, AGS GC cells, which have the best response to polyphyllin I, were used to establish the model. The control (PBS) and drug (polyphyllin I, $0.2 \mathrm{mg} / \mathrm{kg}$ ) groups were also established. Drugs were administrated twice a week while mouse weight and tumor size were measured every 4 days. After 32 days, mice were decapitated under anesthesia, and tumors were weighed and then fixed in paraffin.

As shown in Figure $5 A$, no significant difference in the 
A
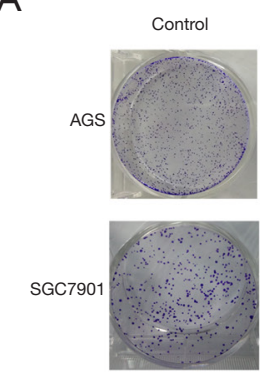

C
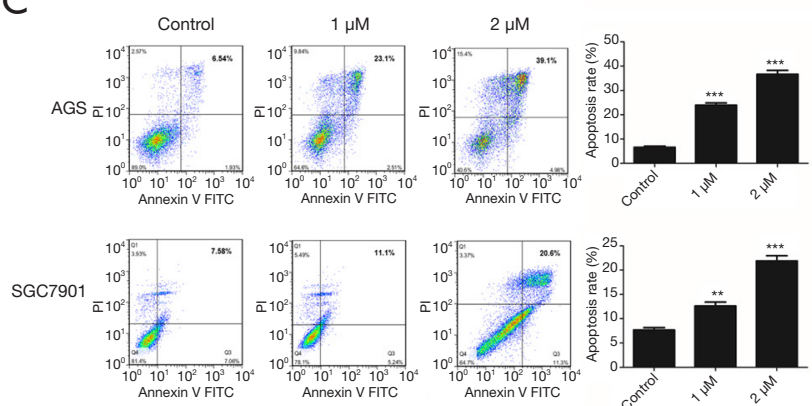

B
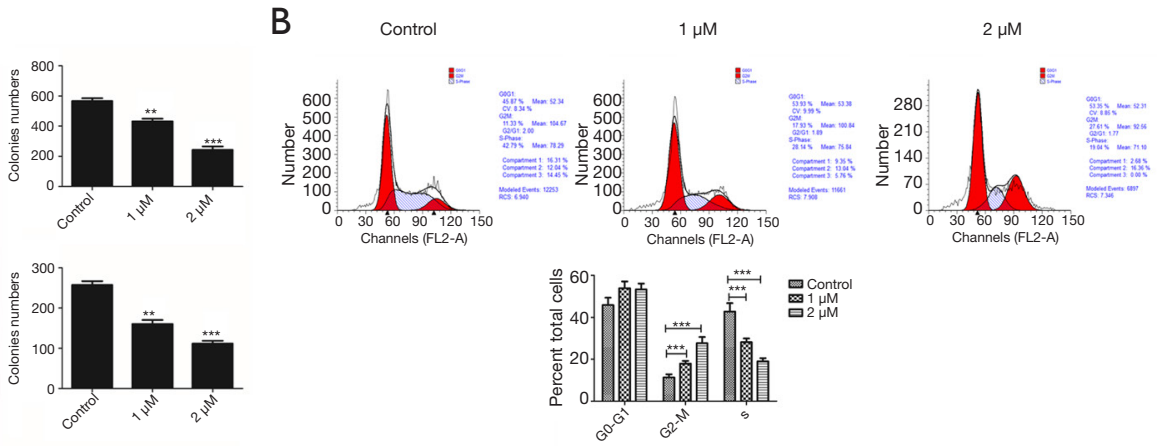

D
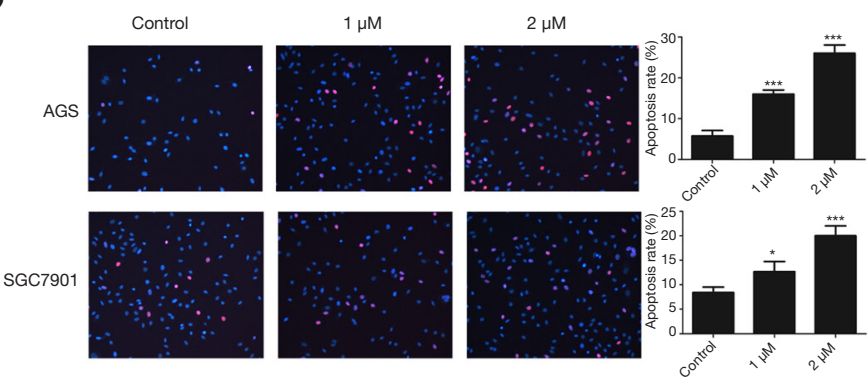

Figure 3 Polyphyllin I inhibit the proliferation and promotes the apoptosis of GC cells in vitro. (A) Effects of polyphyllin I on GC cell colony formation. The colony numbers of two GC cell lines were mounted following treatment with different concentrations of polyphyllin I (1 and $2 \mu \mathrm{M})$ for $48 \mathrm{~h}$; (B) effects of polyphyllin I on GC cell cycle. The cell cycle of the AGS GC cell line was measured following treatment with different concentrations of polyphyllin I (1 and $2 \mu \mathrm{M})$ for $48 \mathrm{~h}$; (C) apoptosis in each group was analyzed by flow cytometry; (D) apoptosis in each group was analyzed by TUNEL assay. Error bars represent the mean \pm SD of three independent experiments. * $\mathrm{P}<0.05$; **, $\mathrm{P}<0.01 ;{ }^{* * *}, \mathrm{P}<0.001$ vs. control group $(\mathrm{n}>3)$.

mouse weight was observed between the drug and control groups. The tumor volume had been growing steadily in both groups, but the growth rate of the drug group was obviously slower than that of the control, indicating that polyphyllin I could inhibit GC growth (Figure 5B,C). The expression level of $\mathrm{Ki}-67$, a proliferation marker of tumor cells, in the drug group transplantable tumor was much lower than that in the control (Figure 5D). Therefore, we concluded that polyphyllin I $(0.2 \mathrm{mg} / \mathrm{kg})$ could inhibit the growth of GC in vivo without obvious toxicity.

\section{Discussion}

Previous studies have shown that natural medicine, such as polyphyllin I could be considered a potential agent for the treatment of human cancers. However, the current data are limited and the exact mechanisms involved in the anticancer activity of polyphyllin I have not yet been elucidated. In the present study, a luciferase screening system was constructed using Bcl-2, a marker of apoptosis, as a reporter gene, and the current study examined whether polyphyllin I can inhibit the activity of GC cells. However, how polyphyllin I affect the $B c l-2$ gene and induces apoptosis is unknown. This study demonstrated significant cell growth inhibition in two GC cell lines, confirming the consistency of our findings and further strengthening our hypothesis.

It was found that polyphyllin I can restrain the IL-6activated JAK2/STAT3 pathway. IL-6 is a widespread cytokine that participates in several biological responses, particularly the JAK signaling pathway (17). Its binding to cell surface receptors results in the activation of the JAKs, which leads to STAT3 phosphorylation. The phosphorylated STAT3 then dimerizes and trans locates to the nucleus, binds with target genes and regulates the DNA transcriptional activity. The expression level of p-JAK2 and p-STAT3 was detected in GC cells treated with IL-6 and polyphyllin I. The results indicated that polyphyllin I can offset the promotion function of IL-6 on p-STAT3. It is known that AG490 mainly inhibits the phosphorylation of JAK2 tyrosine (701) or serine (727), blocking the 

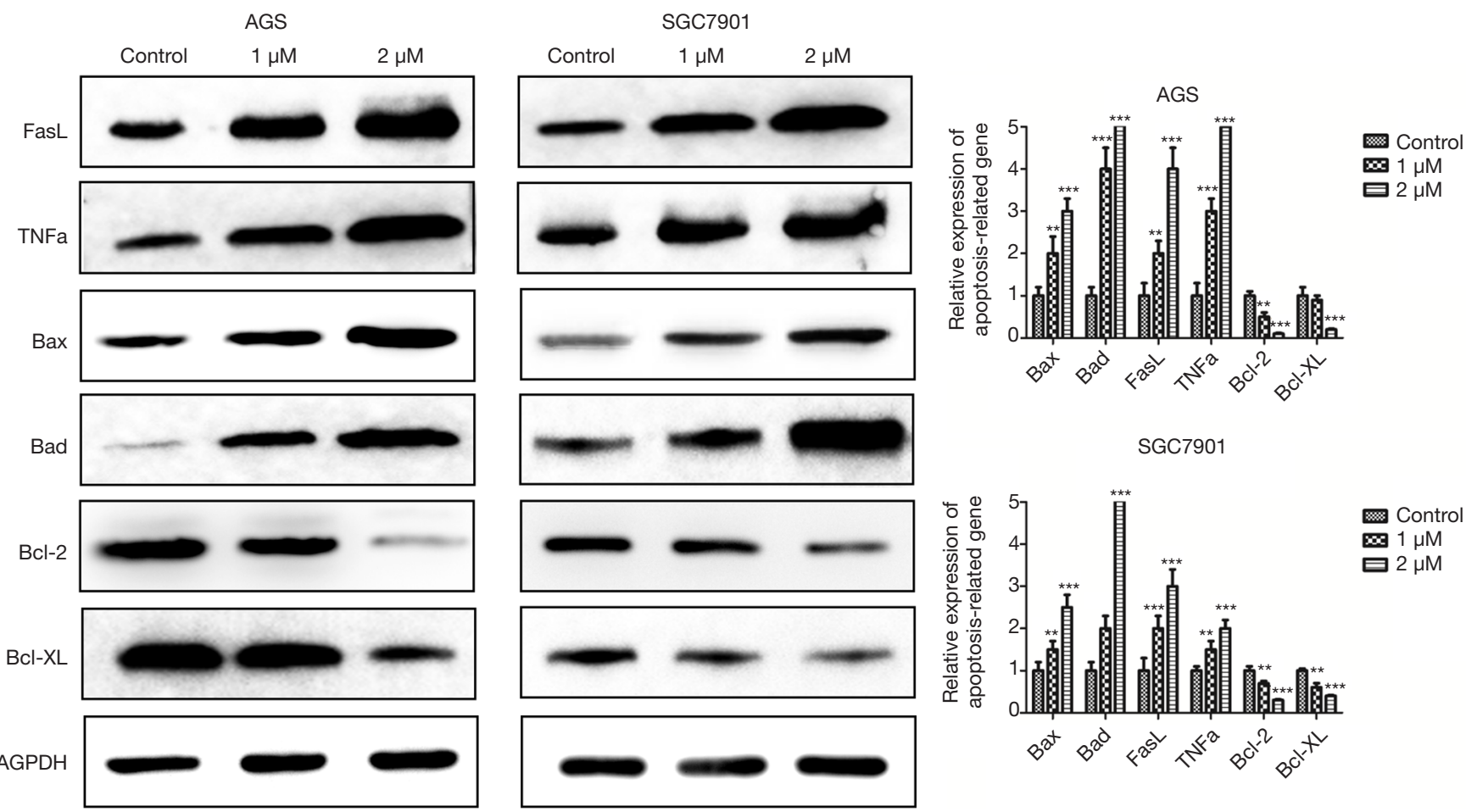

Figure 4 Effects of polyphyllin I on GC cell apoptosis related proteins. Western blot analysis of FasL, TNFa, Bax, Bad, Bcl-2 and Bcl-XL in GC cells treated with polyphyllin I at 1 and $2 \mu \mathrm{M}$ for $48 \mathrm{~h}$. Error bars represent the mean \pm SD of three independent experiments. **, $\mathrm{P}<0.01 ;{ }^{* * *}, \mathrm{P}<0.001$ vs. control group $(\mathrm{n}>3)$.

downstream kinase activation $(18,19)$. The physiological and pathological function of cytokines were then broken, thereby inhibiting tumor growth and transformation. The western blot analysis results showed that the expression pattern of p-JAK2 and p-STAT3 in GC cells treated with AG490 was different from that in cells treated with polyphyllin I. Polyphyllin I inhibited the expression of p-STAT3 but not p-JAK2. These confirmed the speculation that polyphyllin I do not function as a JAK2 inhibitor, but likely as a STAT3 phosphorylation inhibitor. However, all these results were obtained under a low polyphyllin I concentration $(1 \mu \mathrm{M})$. When the concentration was increased, the p-JAK2 expression decreased. The reason for this phenomenon is likely to be the fact that a higher concentration of polyphyllin I strongly inhibits the expression of p-STAT3, leading to the activation of certain negative feedback mechanisms, thus affecting the expression of p-JAK2 and any other protein. This also indirectly proves that the JAK2/STAT3 signaling pathway may be part of a signal loop. In addition, we also detected the effect of the drug on JAK1/STAT1 pathway and obtained results similar to JAK2/STAT3 pathway, which also indicated that the drug had an effect on the entire JAK/STAT pathway.

To date, several STAT3-targeting agents have been identified. These candidate drugs have been shown to work in two different ways. The first is by directly interacting with STAT3 and disturbing STAT3 phosphorylation. This group includes, among others, ST3-H2A2 (20) and S3I-1757 (21). The second is by mainly acting on the upstream protein of STAT3 and indirectly affecting STAT3 activity. Several well-known anti-tumor compounds, such as AZD1480 (22) and AG490 (23), belong to this group. Although all these agents have good potential to inhibit STAT3 activity, very few of them have been used in clinical trials, due to their toxicity and intolerance. So far, there are still no anti-tumor drugs that can act directly on STAT3 protein on the market. However, some STAT3 inhibitors, such as BP-1-102 (24), have been shown to have good in vivo and in vitro activity in preclinical experimental models, which provides hope for the use of STAT3 inhibitors as candidate drugs in clinical trials. In addition, some natural products with a STAT3 inhibition activity have been tested in clinical trials. One such product is curcumin, which is used in the treatment of pancreatic cancer and has 
A

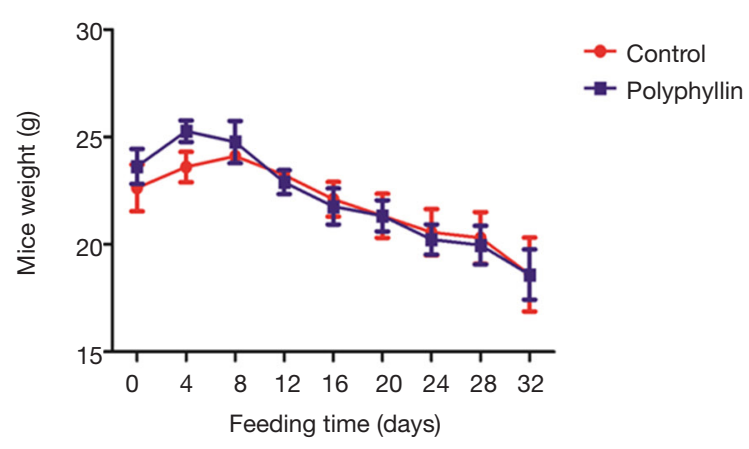

C

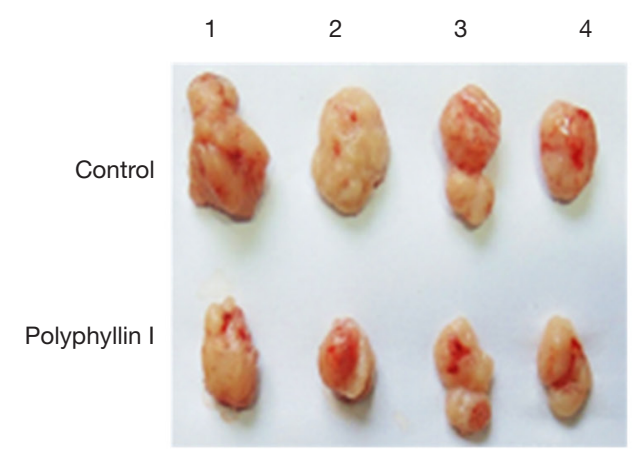

B
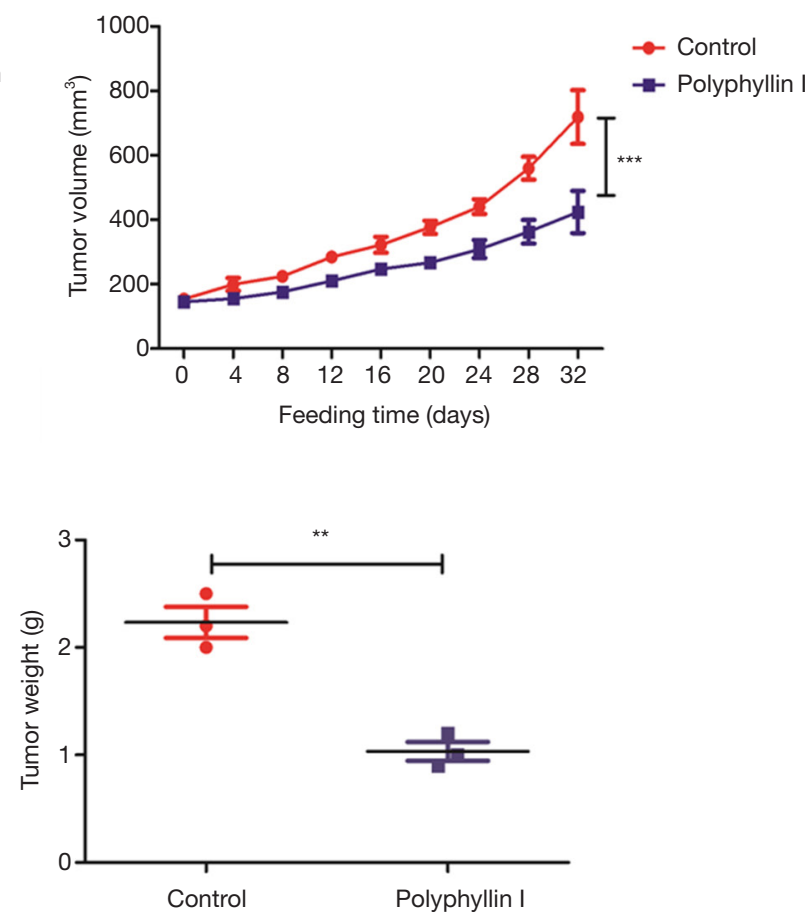

D
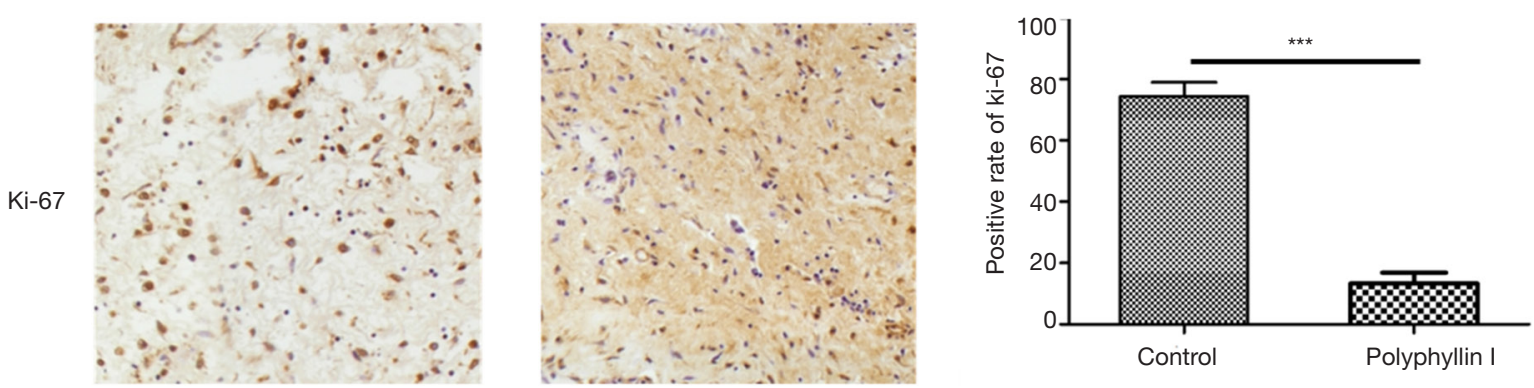

Figure 5 Tumor formation in nude mice. (A) $0.2-\mathrm{mg} / \mathrm{kg}$ polyphyllin I injection did not cause obvious weight loss, as compared with the control group. Error bars represent the mean \pm SD of more than two independent experiments; (B) $0.2-\mathrm{mg} / \mathrm{kg}$ polyphyllin I injection markedly suppressed GC tumor growth; (C) $0.2-\mathrm{mg} / \mathrm{kg}$ polyphyllin I injection decreased the GC tumor weight; (D) immunohistochemistry of Ki-67 in the transplantable tumor (magnification, $\times 200$ ). Error bars represent the mean \pm SD of three independent experiments. **, $\mathrm{P}<0.01 ; * * *, \mathrm{P}<0.001$ vs. control group $(\mathrm{n}>3)$.

entered a phase II clinical trial (NCT00094445), as well as resveratrol, which is used in colon cancer treatment and whose tolerability and pharmacokinetic parameters are also being studied (NCT00433576). Therefore, identifying safe and effective STAT3 inhibitors has become crucial to the development of anti-tumor drugs.
Polyphyllin I, one of the most important components of Rhizoma Paridis, has been proven to be safe and well tolerated, and has been widely used by clinicians in China, from ancient times to the present day. Polyphyllin I have a good anti-inflammatory and anti-tumor activity. In the present study, the possible mechanism through which 
polyphyllin I fights against cancer was revealed for the first time: Polyphyllin I may work as a p-STAT3 inhibitor to selectively depress signaling pathways that STAT3 is involved in, as well as and its downstream apoptosis related genes. A serious of cell experiments in this work have indicated that Polyphyllin I can inhibit the proliferation and promotes the apoptosis of GC cells in vitro. The WB results confirmed that polyphyllin I can both promote death factormediated apoptosis pro-apoptotic factors (FasL, TNFa) and mitochondrial pathway mediated apoptosis pro-apoptotic factors (Bax, Bad). However, the anti-apoptotic factors (bcl-2, bcl-xl) were inhibited. These results showed that polyphyllin I promote the apoptosis of GC cells through the combined action of various apoptotic pathways. The in vivo anti-tumor activity of polyphyllin I demonstrated that Polyphyllin I is low cytotoxic, which improves the feasibility of clinical trials. These findings suggested that polyphyllin I may be a new candidate anti-cancer drug targeting STAT3, a core part of several signaling pathways in GC cells. Further studies should focus on polyphyllin I, as it may lead to the development of more effective and less toxic anti-tumor drugs that can benefit more patients.

\section{Acknowledgments}

Funding: This work was supported by a grant from Project of science and Technology Department of Qinghai Province (No. 2015-ZJ-751), and the National Natural Science Foundation of China (No. 81301898).

\section{Footnote}

Reporting Checklist: The authors have completed the ARRIVE reporting checklist. Available at http://dx.doi. org/10.21037/tcr-20-66

Data Sharing Statement: Available at http://dx.doi. org/10.21037/tcr-20-66

Conflicts of Interest: All authors have completed the ICMJE uniform disclosure form (available at http://dx.doi. org/10.21037/tcr-20-66). The authors have no conflicts of interest to declare.

Ethical Statement: The authors are accountable for all aspects of the work in ensuring that questions related to the accuracy or integrity of any part of the work are appropriately investigated and resolved. Experiments were performed under a project license (NO.:2018-SRFA-088) granted by institutional ethics board of The First Affiliated Hospital of Nanjing Medical University, in compliance with Nanjing Medical University institutional guidelines for the care and use of animals.

Open Access Statement: This is an Open Access article distributed in accordance with the Creative Commons Attribution-NonCommercial-NoDerivs 4.0 International License (CC BY-NC-ND 4.0), which permits the noncommercial replication and distribution of the article with the strict proviso that no changes or edits are made and the original work is properly cited (including links to both the formal publication through the relevant DOI and the license). See: https://creativecommons.org/licenses/by-nc-nd/4.0/.

\section{References}

1. Siegel RL, Miller KD, Jemal A. Cancer statistics, 2018. CA Cancer J Clin 2018;68:7-30.

2. Mai HN, Chung YH, Shin EJ, et al. IL-6 knockout mice are protected from cocaine-induced kindling behaviors; possible involvement of JAK2/STAT3 and PACAP signalings. Food Chem Toxicol 2018;116:249-63.

3. Singh AK, Bhadauria AS, Kumar U, et al. Novel Indolefused benzo-oxazepines (IFBOs) inhibit invasion of hepatocellular carcinoma by targeting IL-6 mediated JAK2/STAT3 oncogenic signals. Sci Rep 2018;8:5932.

4. Kang DY, Sp N, Kim DH, Jet al. Salidroside inhibits migration, invasion and angiogenesis of MDA-MB 231 TNBC cells by regulating EGFR/Jak2/STAT3 signaling via MMP2. Int J Oncol 2018;53:877-85.

5. Wang M, Lin T, Wang Y, et al. CXCL12 suppresses cisplatin-induced apoptosis through activation of JAK2/ STAT3 signaling in human non-small-cell lung cancer cells. Onco Targets Ther 2017;10:3215-24.

6. Liu FY, Safdar J, Li ZN, et al. CCR7 regulates cell migration and invasion through JAK2/STAT3 in metastatic squamous cell carcinoma of the head and neck. Biomed Res Int 2014;2014:415375.

7. Onimoe GI, Liu A, Lin L,et al. Small molecules, LLL12 and FLLL32, inhibit STAT3 and exhibit potent growth suppressive activity in osteosarcoma cells and tumor growth in mice. Invest New Drugs 2012;30:916-26.

8. Giraud AS, Menheniott TR, Judd LM. Targeting STAT3 in gastric cancer. Expert Opin Ther Targets 2012;16:889-901.

9. Shan Y, Gnanasambandan K, Ungureanu D, et 
al. Molecular basis for pseudokinase-dependent autoinhibition of JAK2 tyrosine kinase. Nat Struct Mol Biol 2014;21:579-84.

10. Bogani C, Bartalucci N, Martinelli S, et al. mTOR inhibitors alone and in combination with JAK2 inhibitors effectively inhibit cells of myeloproliferative neoplasms. PLoS One 2013;8:e54826.

11. Verstovsek S, Mesa RA, Gotlib J, et al. Efficacy, safety, and survival with ruxolitinib in patients with myelofibrosis: results of a median 3-year follow-up of COMFORT-I. Haematologica 2015;100:479-88.

12. Li GB, Fu RQ, Shen HM, et al. Polyphyllin I inducesmitophagic and apoptotic cell death in human breast cancer cells by increasing mitochondrial PINK1 levels. Oncotarget 2017;8:10359-74.

13. Chang J, Li Y, Wang X, et al. Polyphyllin I suppresses human osteosarcoma growth by inactivation of Wnt/ $\beta$-catenin pathway in vitro and in vivo. Sci Rep 2017;7:7605.

14. Liu J, Man S, Li J, et al. Inhibition of diethylnitrosamineinduced liver cancer in rats by Rhizoma paridis saponin. Environ Toxicol Pharmacol 2016;46:103-9.

15. He H, Sun YP, Zheng L, et al. Steroidal saponins from Paris polyphylla induce apoptotic cell death and autophagy in A549 human lung cancer cells. Asian Pac J Cancer Prev 2015;16:1169-73.

16. Xiao T, Zhong W, Zhao J, et al. Polyphyllin I suppresses the formation of vasculogenic mimicry via Twist1/VEcadherin pathway. Cell Death Dis 2018;9:906.

Cite this article as: Han J, Kan J, Wang X, Yuan Q, Song X, Huang M, Wu H. Polyphyllin I suppresses proliferation and promotes apoptosis of gastric cancer cell by inhibiting stat 3 phosphorylation. Transl Cancer Res 2020;9(8):4715-4725. doi: $10.21037 /$ tcr-20-66
17. Heinrich PC, Behrmann I, Haan S, et al. Principles of interleukin (IL)-6-type cytokine signalling and its regulation. Biochem J 2003;374:1-20.

18. Joung YH, Na YM, Yoo YB, et al. Combination of AG490, a Jak2 inhibitor, and methylsulfonylmethane synergistically suppresses bladder tumor growth via the Jak2/STAT3 pathway. Int J Oncol 2014;44:883-95.

19. Luo C, Laaja P. Inhibitors of JAKs/STATs and the kinases: a possible new cluster of drugs. Drug Discov Today 2004;9:268-75.

20. Timofeeva OA, Tarasova NI, Zhang X, et al. STAT3 suppresses transcription of proapoptotic genes in cancer cells with the involvement of its N-terminal domain. Proc Natl Acad Sci U S A 2013;110:1267-72.

21. Zhang X, Sun Y, Pireddu R, et al. A novel inhibitor of STAT3 homodimerization selectively suppresses STAT3 activity and malignant transformation. Cancer Res 2013;73:1922-33.

22. Hedvat M, Huszar D, Herrmann A, et al. The JAK2 inhibitor AZD1480 potently blocks Stat3 signaling and oncogenesis in solid tumors. Cancer Cell 2009;16:487-97.

23. Huang C, Cao J, Huang KJ, et al. Inhibition of STAT3 activity with AG490 decreases the invasion of human pancreatic cancer cells in vitro. Cancer Sci 2006;97:1417-23.

24. Zhang X, Yue P, Page BD, et al. Orally bioavailable smallmolecule inhibitor of transcription factor Stat 3 regresses human breast and lung cancer xenografts. Proc Natl Acad Sci U S A 2012;109:9623-8. 